\title{
Study of the performance of prototypes of straw tube tracker by measuring cosmic rays
}

\author{
Akshay Malige $^{1, *}$, Grzegorz Korcyl ${ }^{1}$, and Narendra Rathod ${ }^{1}$ \\ ${ }^{1}$ The Marian Smoluchowski Institute of Physics, Jagiellonian University, Lojasiewicza 11, 30-348 \\ Kraków, Poland
}

\begin{abstract}
Straw tube detector developed for the PANDA experiment in [1], will be used for tracking and identifcation of charged particles in the Forward Tracker (FT). The detector read-out will be incorporated in PANDA DAQ running in trigger-less mode by means of Synchronization Of Data Acquisition Network (SODAnet). SODAnet is the protocol used to synchronize individual detector subsystems by providing a common clock signal and timestamps. The reconstruction of events out of many fragments is done with the Burst Building Network. The first tests of such system have been performed with prototypes of FT and ElectroMagnetic Calorimeter modules (EMC) in [1] measuring cosmic rays. Those tests allow to evaluate the detectors as well as the synchronization and processing systems. The reconstruction of particle tracks has been developed and evaluated. The results on the track reconstruction, spatial resolution and energy loss via Time over Threshold (TOT) method is described together with the DAQ performance.
\end{abstract}

\section{Prototype of PANDA Forward Tracker}

Straw tubes which are cylindrical mini drift chambers are the building blocks of PANDA FT in [3]. The tubes are filled with a gas mixture of $90 \% \mathrm{Ar}$ and $10 \% \mathrm{CO}_{2}$ at 2 bars pressure and contain $20 \mu \mathrm{m}$ gold plated tungsten anode wire stretched along the cylinder axis. The wall of the straw tube is made of aluminized Mylar foil of $27 \mu \mathrm{m}$ thickness. The length of the tubes is $150 \mathrm{~cm}$ and has a diameter of $1.01 \mathrm{~cm}$. The prototype of the FT built at the Jagiellonian University, Krakow, consists of 256 straws arranged in four double layer of straws i.e. two horizontal and two vertical. Two double layers were mounted in separate frames and each double layer consisted of two modules each with 32 straws. Modules are read-out by two Front End boards (FE), each consisting of two PASTTREC ASIC's in [5] featuring analog signal shaping circuit and a leading edge discriminator. FE are connected to a Trigger and Read-out Board (TRB) developed by the HADES collaboration in [6] which performs TimeDigit-Conversion and data transmission over a $2 \mathrm{~Gb}$ optical link. The data processing is described in more details below.

\section{PANDA DAQ}

The expected event rates at PANDA are in the order $2 \times 10^{7} \mathrm{~s}^{-1}$ and this results in the data flow from detectors at the level of $200 \mathrm{~GB} / \mathrm{s}$ which is has to be suppressed by 2-3 orders of

\footnotetext{
*e-mail: mailme.akshaym@gmail.com
} 
magnitude by a dedicated on-line event processing algorithms running on a computer farm. In order to reconstruct complete event out of many fragments provided by read-out modules, these need to be marked with exact time stamp. Specially for this purpose the SODAnet was designed. It is a protocol developed to distribute time information to all sub-detector systems of the PANDA spectrometer. The distribution is done in two ways. First of all the packets containing time-stamp information are sent to each Data Concentrator (DC) merging data from the all detector read-out modules in [4]. Secondly, the clock signal, from the transmission, is recovered and it is used for DC logic synchronization. Such an approach assures that the DC's and FE are synchronized to the same clock for all the PANDA units. The reconstruction of events out of many fragments is done with the Burst Building Network. These events are later processed and filtered in the compute farm before getting sent to the storage.

\section{Experimental setup and measurements}

A prototype of such DAQ architecture has been set-up with two detector subsystems i.e. FT and the EMC and has been tested for the first time measuring cosmic rays. The EMC played the role of a reference detector because it was placed $\approx 1 \mathrm{~m}$ behind the FT. The FT delivers analog signals which are shaped and discriminated by the FE. The digital signal which comes from the leading edge discriminator is converted to time by the TDC implemented in FPGA. Two TRB's were used in the present prototype with total 10 FPGAs altogether: 5 programmed as TDCs, one as EMC DC, one as SODAnet source and one as TRB DC. Three $\mu$ TCA's compliant boards equipped with a Xilinx Virtex 5 FX70T -2 FPGA, 4 GB DDR2 RAM, $1 \mathrm{~Gb}$ Ethernet, are used for the Compute Node in [2] $(\mathrm{CN})$ and receives data from the DC of the TRB.

The burst building has been split into three parts, CN1, CN2 and CN3 respectively. CN1 receives data from EMC DC and forwards to $\mathrm{CN} 3$ through backplane, $\mathrm{CN} 2$ receives and merges data from the FT TRB1 and TRB2, forwards to $\mathrm{CN} 3$ through backplane. $\mathrm{CN} 3$ receives and merges data from $\mathrm{CN} 1$ and $\mathrm{CN} 2$, builds events and sends out to storage through Gigabit Ethernet.

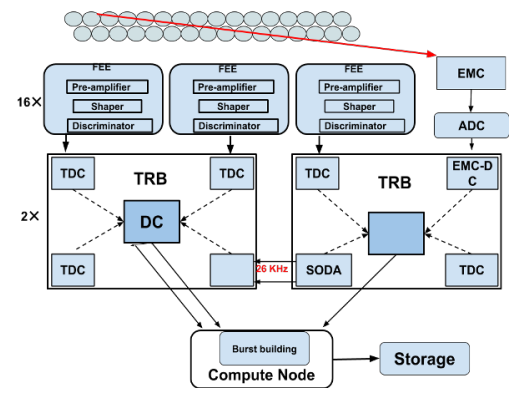

(a)

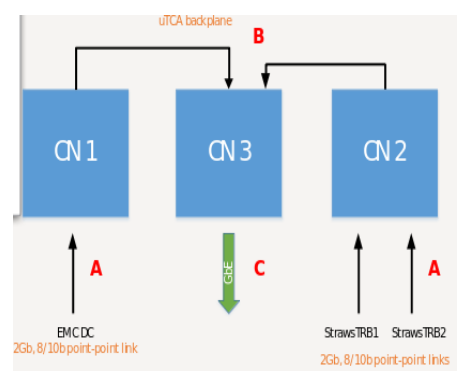

(b)

Figure 1: (a)-The general view of the DAQ architecture. The FEE is connected to the Data Concentrators which receive packets from SODAnet. and (b) Schematic representation of the compute node design. 


\section{Results and conclusions}

Cosmic data set was collected for 56 hours and analysed for track reconstruction. Only events with time correlation between EMC and all layers of FT were selected and the EMC was used as T0 for drift time calculation resulting in a range of about $\approx 200 \mathrm{~ns}$, as expected from previous measurements in [4]. Drift radius calibration was calculated assuming uniform illumination of the straw tubes with $R=0.505 \mathrm{~cm}$ and maximum drift time of $200 \mathrm{~ns}$. Linearly fitted track candidates were used to determine the spatial resolution of the system which was $359 \mu \mathrm{m}$. Previous measurements provide 2 times better resolution. The difference is due to better time alignment channels and the improved calibration is now in progress.

The prototype of the DAQ that includes all parts of the foreseen readout system that is: independent subsystems, synchronization network and burst building facility has been constructed and run successfully. Track reconstruction method, that includes data from both subsystems, show the long term stability and synchronization of the readout electronics which was the main goal of the test.

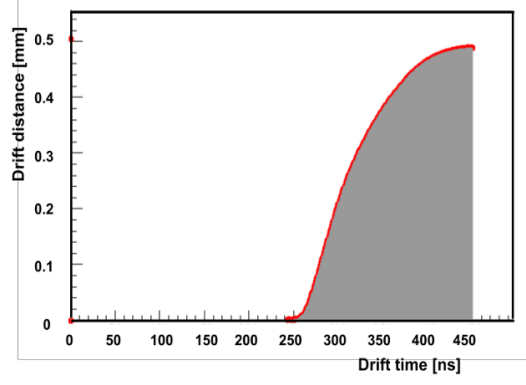

(a)

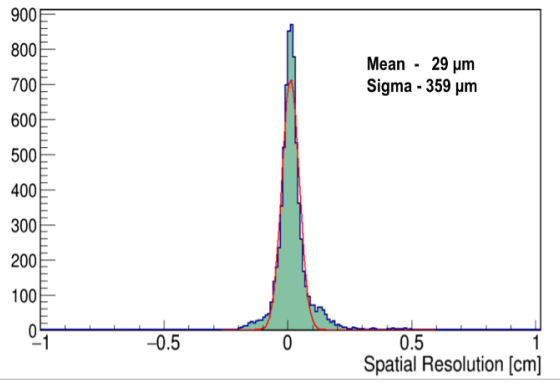

(b)

Figure 2: (a) Calibration curve representing the correlation between the drift time and drift radius and (b) Spatial resolution from 256 straws measured for cosmic rays being $359 \mu \mathrm{m}$.

\section{Acknowledgement}

This project has received funding from the European Union's Horizon 2020 research and innovation programme under the Marie Sklodowska-Curie grant agreement No - 665778 National Science center, Poland 2016/23/P/St2/04066 POLONEZ and by Ministry of Science and Higher Education 7150/E$338 / \mathrm{M} / 2018$.

\section{References}

[1] PANDA Collaboration, letter of intent for PANDA - Strong Interaction Studies with Antiprotons, Technical report FAIR- ESAC (2004)

[2] G. Korcyl et al., IEEE Trans. Nucl. Sci. 65, 821-827 (2017)

[3] J. Smyrski et al., JINST 12, C06032 (2017)

[4] P. Strzempek, Development and evaluation of a signal analysis and a readout system of straw tube detectors for the PANDA spectrometer, Doctoral thesis (2017)

[5] D. Przyborowski et al., JINST 11, P08009 (2016) 